Bangladesh J. Plant Taxon. 25(1): 79-91, 2018 (June)

(C) 2018 Bangladesh Association of Plant Taxonomists

\title{
TRADITIONAL HONEY PRODUCTION AND BEE FLORA OF ESPIYE, TURKEY
}

\author{
Mustafa Karaköse, Ridvan Polat ${ }^{1}$, M. Oliur Rahman ${ }^{2}$ and UĞUR ÇakilcioĞlu ${ }^{3}$ \\ Giresun University, Espiye Vocational School, Giresun, Turkey \\ Keywords: Bee flora; Honeybee; Espiye; Turkey.
}

\begin{abstract}
This paper presents potential honey bee plants in Espiye (Giresun) which can be considered as a guide for beekeepers and researchers. A total of 149 taxa belonging to 125 genara and 48 families were recorded as pollen and nectary sources for honey bee colonies at Espiye (Giresun) region. Among the recorded taxa 58 were Phanerophytes, 57 taxa Hemicryptophytes, 19 taxa Therophytes, 13 taxa Cryptophytes and 2 taxa Chamaephytes. Updated nomenclature along with the families, local names, life form, flowering period and ecological status have been furnished under 94 herbs, 28 shrubs and 27 trees.
\end{abstract}

\section{Introduction}

Turkey is one of the countries where the honey production is at the highest level in the World (Özturk and Erkan, 2010). In the recent past, the forest area in Turkey has increased from 20.2 million/ ha to $22.3 \mathrm{million} / \mathrm{ha}$ between 1973 and 2015 (OGM, 2013-2015). Very recently, the Forest General Directorate has started to implementing the honey action plan to promote honey production and contribute to rural development (OGM, 2013-2015). As part of the action plan, up to 356 honey forests have been established and now, Turkey is in the second row in the world's honey production and beekeeping. Turkey produces $92 \%$ of the world's pine honey, specifically in its West Mediterranean and South Aegean regions. In Turkey, there are 57,000 registered beekeepers and 6.6 million registered hives as of 2014 (Duyum and Friedman, 2015). Siral1 (2009) reported about 300 species of natural or cultivated nectar plants from Turkey comprising about $75 \%$ of the nectar plant species in the world.

The botanic origin of honey is one of the most important parameters of honey quality (Tucak et al., 2000, 2004). The taste, smell and colour of honey are changed according to the nectar of the flowers. In nature, bees visit flowers to produce honey and to take food and they collect pollen, nectar or both from plants during their visit to plants. Nectar is found in the special part of some flowers or in other organs (stem, leaves etc.). Bees visit definite organs of some herbacous and lingeous plants to collect components of honey. Therefore, bee plants can not be determined only by palynologcal studies in honey (Tew, 1998; Tutkun, 2000).

Turkey is the home of three phyto-geographic regions (Euro-Siberian, Mediterranean and Irano-Turanian) in terms of plant geography. Each floristic region has its own plant composition and this affects the variety, quality and authenticity of the produced honey. Furthermore, honey production in Turkey has increased to 114.471 tonnes from 54.655 tonnes in the period from 1991 to 2017 (TUİK, 2018). However, no studies on bee plants have been made in Espiye, Giresun so far. Proper taxonomic identity of bee plants of the country, more particularly in Espiye is lacking.

\footnotetext{
${ }^{1}$ Bingöl University, Genç Vocational School, Bingöl, Turkey.

${ }^{2}$ Department of Botany, University of Dhaka, Dhaka 1000, Bangladesh.

${ }^{3}$ Munzur University, Pertek Sakine Genç Vocational School, Tunceli, Turkey. Corresponding author. Email: ucakilcioglu@yahoo.com
} 
Therefore, the present study aimed at producing a bee flora of Espiye, Giresun and preparing a database which will not only contribute to honey producers but also contribute greatly to the determination of honey contents.

\section{Materials and Methods}

Espiye (Giresun) is situated in the Eastern Black Sea Region (costal and inner parts), surrounded by the Gorele and Tirebolu in the east, Güce and Alucra in the South, Yağlidere and Keşap in the west and by Black Sea in the North (Fig. 1). Espiye is located between $140^{\circ} 44^{\prime} 14^{\prime \prime}$ to $40^{\circ} 58^{\prime} 27^{\prime \prime} \mathrm{N}$ and $38^{\circ} 37^{\prime} 11^{\prime \prime}$ to $38^{\circ} 48^{\prime} 39^{\prime \prime} \mathrm{E}$ with an area of c. 21,100 ha. The altitude of the study area is $1600 \mathrm{~m}$ asl. Approximately 2,500 species have been recorded in the region and this region hosts about $28 \%$ of Turkey's flora (Dokap, 2000). Since there is no meteorological station in Espiye, the climatic data of Giresun were taken into consideration. The annual average rainfall of Giresun is $1,288.4 \mathrm{~mm}$ and the average temperature is $14.6^{\circ} \mathrm{C}$ (Table 1). The climate type of the area is humid based on Thornthwaite climate classification (TSMS, 2017).
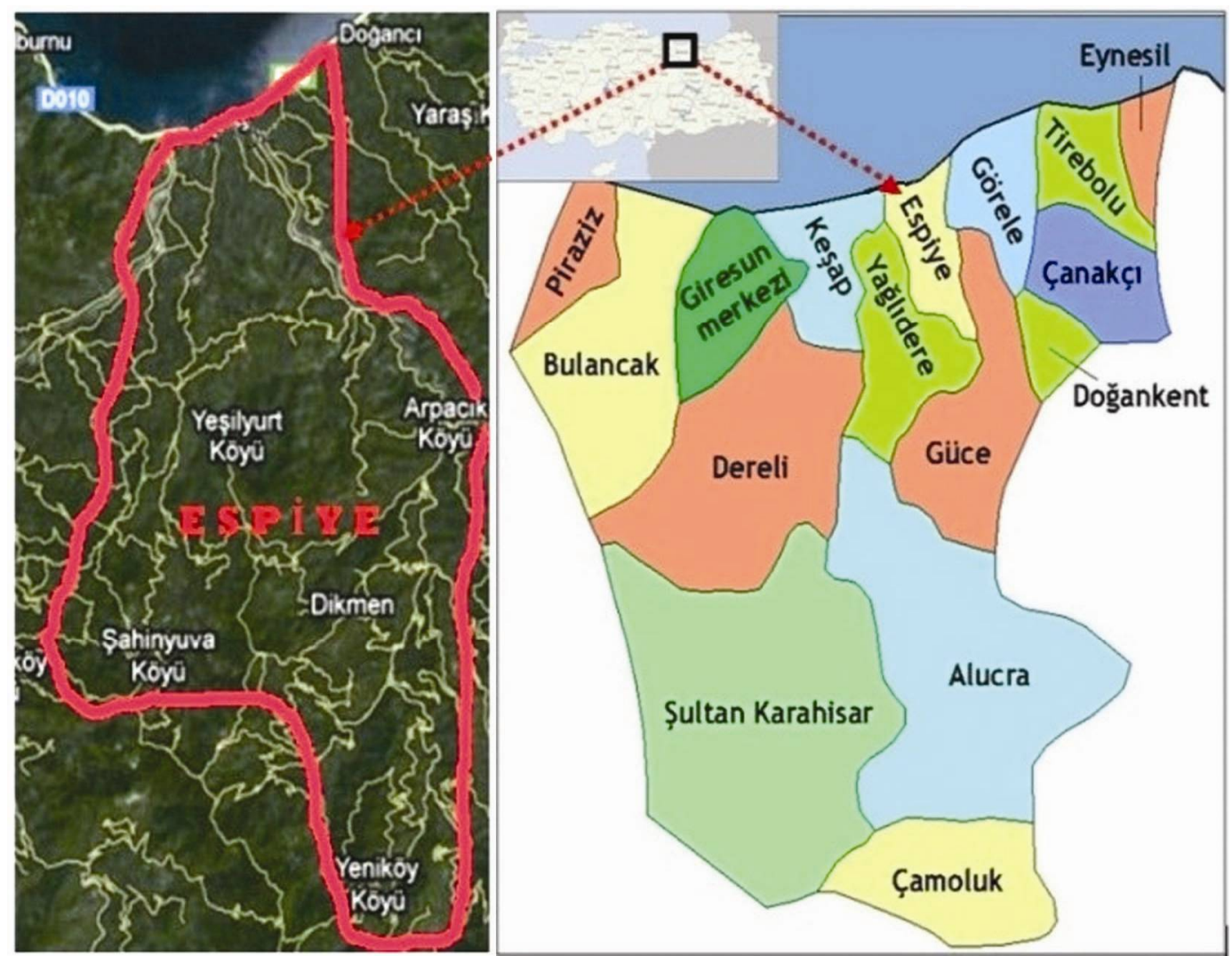

Fig. 1. Map of the study area Espiye, Giresun.

Extensive field surveys were conducted over four years from 2012 to 2016 in the villages and rural areas of Espiye (Giresun) region in different seasons. Field visits were made along with beekeepers and a total of 17 beekeepers over middle age were interviewed to collate information on the bee plants. Abundance and density of the bee and bee plant populations were observed in 
the field. Plant samples were collected from the field and the collected specimens were critically studied and identified. Identifications were confirmed using the Flora of Turkey and the East Aegean Islands, and List of Turkish Plants (Davis, 1965-1985; Davis et al., 1988; Güner et al., 2000, 2012). Life forms of these plants were identified according to the Raunkiaer's system (Raunkiaer, 1937). The families are arranged alphabetically and the taxa under each family are placed in an alphabetical order. The voucher specimens have been preserved in Giresun University Herbarium.

Table 1. Average climate values of Giresun Meteorology Station (1929-2016).

\begin{tabular}{lccccccccccccc}
\hline Months & Jan & Feb & Mar & Apr & May & Jun & Jul & Aug & Sep & Oct & Nov & Dec & Mean \\
\hline Temperature $\left({ }^{\circ} \mathrm{C}\right)$ & 7.2 & 7.1 & 8.0 & 11.3 & 15.5 & 20.1 & 22.7 & 23.1 & 22.0 & 16.2 & 12.6 & 9.4 & 14.6 \\
Rainfall $(\mathrm{mm})$ & 127.5 & 101.5 & 97.5 & 76.1 & 66.8 & 77.5 & 79.4 & 89.5 & 129.2 & 164.7 & 151.9 & 126.8 & $1,288.4$ \\
\hline
\end{tabular}

\section{Result and Discussion}

A total of 149 plant taxa belonging to 125 genera and 48 families were identified as bee plants (Table 2). Among the identified taxa herbs are represented by 94 taxa, shrubs by 28 and trees by 27 taxa. Asteraceae and Fabaceae are the largest families represented by 17 taxa each, followed by Rosaceae with 15 taxa, Lamiaceae with 12 taxa and Ericaceae with 6 taxa (Fig. 2).

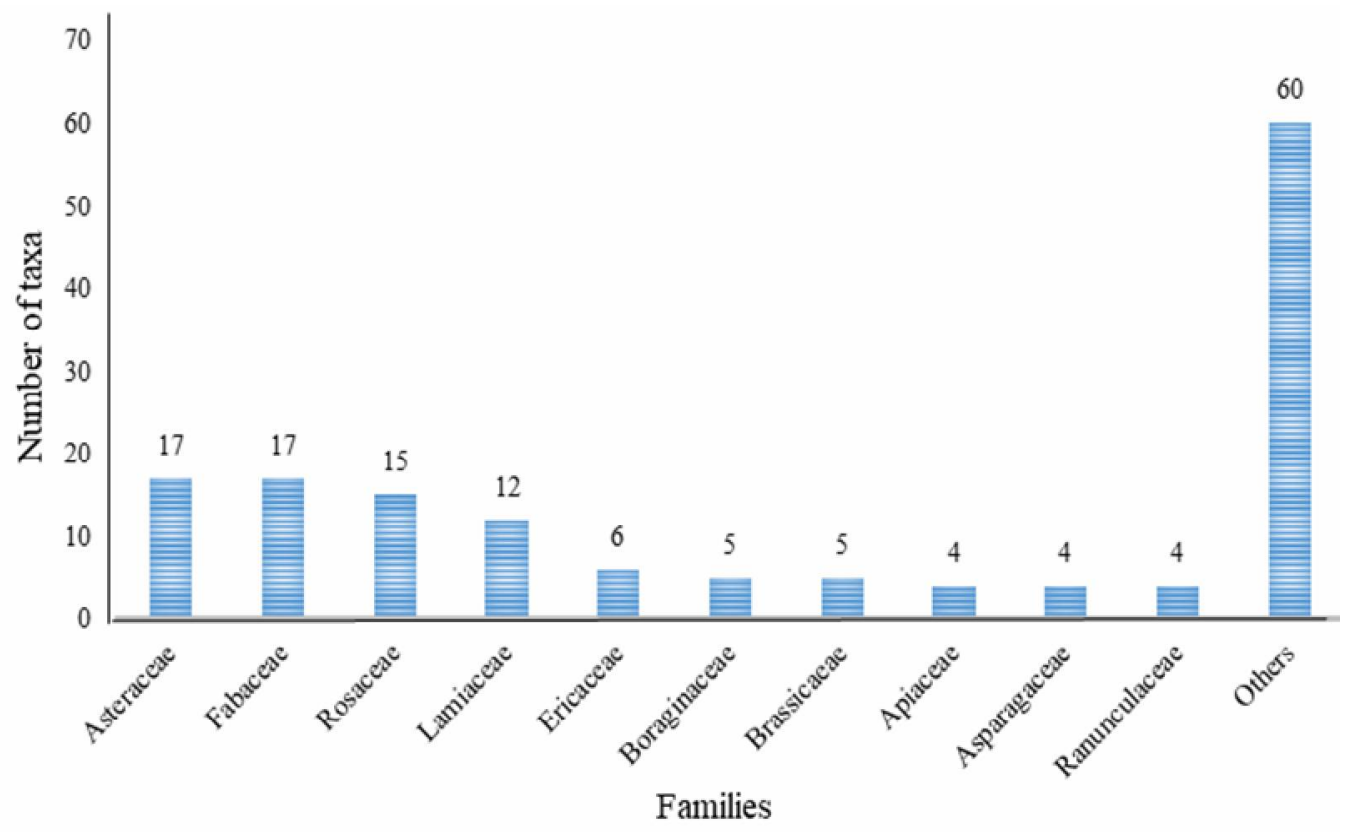

Fig. 2. Distribution of bee plants according to family showing the number of taxa.

The present study revealed that in the study area, 58 taxa are Phanerophytes, 57 taxa Hemicryptophytes, 19 taxa Therophytes, 13 taxa Cryptophytes and 2 taxa are Chamaephytes. 
Table 2. Bee plants of Espiye region along with Turkish name, life form, flowering period, status and vouchers.

\begin{tabular}{|c|c|c|c|c|c|}
\hline Name of the taxa & Turkish name & $\begin{array}{l}\text { Life } \\
\text { form }\end{array}$ & $\begin{array}{l}\text { Flowering } \\
\text { period }\end{array}$ & Status & $\begin{array}{l}\text { Voucher } \\
\text { specimen }\end{array}$ \\
\hline \multicolumn{6}{|l|}{ Adoxaceae } \\
\hline $\begin{array}{l}\text { Sambucus nigra } \mathrm{L} . \\
\text { Aizoaceae }\end{array}$ & Ağaç mürver & $\mathrm{Ph}$ & $4-7$ & Natural & MK 939 \\
\hline $\begin{array}{l}\text { Mesembryanthemum cordifolium } \mathrm{L} \text {. f. } \\
\text { Apiaceae }\end{array}$ & Buz çiçeği & $\mathrm{Ch}$ & $5-9$ & Ornamental & MK 904 \\
\hline Daucus carota $\mathrm{L}$. & Yabani havuç & $\mathrm{H}$ & $4-8$ & Natural & MK 857 \\
\hline Foeniculum vulgare Mill. & Rezene & $\mathrm{H}$ & $5-9$ & Natural & MK 870 \\
\hline Pimpinella anisum $\mathrm{L}$. & Anason & Th & $6-8$ & Cultivation & MK 913 \\
\hline $\begin{array}{l}\text { Smyrnium olusatrum L. } \\
\text { Araliaceae }\end{array}$ & Deli kereviz & $\mathrm{H}$ & $3-5$ & Natural & MK 943 \\
\hline $\begin{array}{l}\text { Hedera helix } \mathrm{L} . \\
\text { Asparagaceae }\end{array}$ & Duvar sarmaşı̆̆ & $\mathrm{Ch}$ & $8-9$ & Natural & MK 875 \\
\hline $\begin{array}{l}\text { Hyacinthus orientalis L. subsp. } \\
\text { orientalis }\end{array}$ & Sümbül & $\mathrm{Cr}$ & $3-5$ & Ornamental & MK 878 \\
\hline Muscari armeniacum Leich. ex Baker & Gavurbaşı & $\mathrm{Cr}$ & $3-5$ & Natural & MK 905 \\
\hline $\begin{array}{l}\text { Ornithogalum oligophyllum E.D. } \\
\text { Clarke }\end{array}$ & Kurt soğanı & $\mathrm{Cr}$ & $4-7$ & Natural & MK 908 \\
\hline $\begin{array}{l}\text { Scilla bifolia } \mathrm{L} \text {. } \\
\text { Asteraceae }\end{array}$ & Orman sümbülü & $\mathrm{Cr}$ & $3-6$ & Natural & MK 940 \\
\hline Artemisia vulgaris L. & Kaba yavşan & $\mathrm{H}$ & $6-9$ & Natural & MK 828 \\
\hline Bellis perennis L. & Koyungözü & $\mathrm{H}$ & $3-8$ & Natural & MK 825 \\
\hline Bidens tripartita $\mathrm{L}$. & Üç suketeni & Th & $7-9$ & Naturalized & MK 830 \\
\hline Calendula officinalis L. & Aynısafa & Th & $1-6$ & Ornamental & MK 832 \\
\hline Cichorium inthybus L. & Hindiba & $\mathrm{H}$ & $4-9$ & Natural & MK 842 \\
\hline Cirsium arvense (L.) Scop. & Köygöçüren & $\mathrm{H}$ & $5-10$ & Natural & MK 843 \\
\hline Cosmos bipinnatus Cav. & Meksika Yıldızı & Th & $6-10$ & Ornamental & MK 851 \\
\hline Cota tinctoria (L.) J. Gay & Boyacı papatyası & $\mathrm{H}$ & $6-7$ & Natural & MK 852 \\
\hline Dimorphotheca ecklonis DC. & Bodrum papatyas 1 & $\mathrm{H}$ & $4-6$ & Ornamental & MK 859 \\
\hline Erigeron aпnuиs (L.) Pers. & Hemşin şifaotu & Th & $6-9$ & Naturalized & MK 866 \\
\hline Eupatorium cannabinum L. & Koyuntırpağı & $\mathrm{H}$ & $7-10$ & Natural & MK 869 \\
\hline $\begin{array}{l}\text { Helichrysum plicatum DC. subsp. } \\
\text { plicatum }\end{array}$ & Mantuvar & $\mathrm{H}$ & $6-8$ & Natural & MK 876 \\
\hline $\begin{array}{l}\text { Lapsana communis L. subsp. } \\
\text { intermedia (M. Bieb.) Hayek }\end{array}$ & Şebrek & $\mathrm{H}$ & $5-10$ & Natural & MK 888 \\
\hline $\begin{array}{l}\text { Solidago virgaurea } \mathrm{L} \text {. subsp. } \\
\text { virgaurea }\end{array}$ & Altınbaşak çiçeği & $\mathrm{H}$ & $7-9$ & Natural & MK 944 \\
\hline $\begin{array}{l}\text { Sonchus asper (L.) Hill subsp. } \\
\text { glaucescens (Jord.) Ball. }\end{array}$ & Gevirtlek & $\mathrm{H}$ & $3-8$ & Natural & MK 945 \\
\hline Taraxacum laxum Hagl. & Gevşek çıtlık & $\mathrm{H}$ & $4-8$ & Natural & MK 949 \\
\hline $\begin{array}{l}\text { Tussilago farfara } \mathrm{L} . \\
\text { Begoniaceae }\end{array}$ & Öksürükotu & $\mathrm{Cr}$ & $3-4$ & Natural & MK 956 \\
\hline $\begin{array}{l}\text { Begonia cucullata Willd. } \\
\text { Berberidaceae }\end{array}$ & Bahçe begonyası & $\mathrm{H}$ & $7-1$ & Ornamental & MK 827 \\
\hline $\begin{array}{l}\text { Berberis vulgaris } \mathrm{L} . \\
\text { Betulaceae }\end{array}$ & Kızılkaramuk & $\mathrm{Ph}$ & $5-6$ & Natural & MK 829 \\
\hline $\begin{array}{l}\text { Alnus glutinosa (L.) Gaertn. subsp. } \\
\text { barbata (C.A. Mey.) Yalt. }\end{array}$ & Kızılağaç & $\mathrm{Ph}$ & $2-4$ & Natural & MK 823 \\
\hline
\end{tabular}


Table 2 (Contd.)

\begin{tabular}{|c|c|c|c|c|c|}
\hline Name of the taxa & Turkish name & $\begin{array}{l}\text { Life } \\
\text { form }\end{array}$ & $\begin{array}{c}\text { Flowering } \\
\text { period }\end{array}$ & Status & $\begin{array}{l}\text { Voucher } \\
\text { specimen }\end{array}$ \\
\hline Corylus avellana L. var. avellana & Findık & $\mathrm{Ph}$ & $2-3$ & Natural & MK 850 \\
\hline C. maxima Mill. & Tombul findik & $\mathrm{Ph}$ & 3 & Cultivation & MK 849 \\
\hline $\begin{array}{l}\text { Bignoniaceae } \\
\text { Campsis radicans (L.) Seem. } \\
\text { Boraginaceae }\end{array}$ & Acem borusu & $\mathrm{Ph}$ & $4-7$ & Ornamental & MK 836 \\
\hline Cynoglossum creticum Mill. & Pisiktetiği & $\mathrm{H}$ & $3-7$ & Natural & MK 856 \\
\hline Echium angustifolium Mill. & Agres & $\mathrm{H}$ & $3-8$ & Natural & MK 863 \\
\hline E. vulgare L. subsp. vulgare & Engerek otu & $\mathrm{H}$ & $5-9$ & Natural & MK 862 \\
\hline $\begin{array}{l}\text { Myosotis laxa Lehm. subsp. } \\
\text { caespitosa (Schultz) Hyl. ex Nordh. }\end{array}$ & Hüthütgözü & $\mathrm{H}$ & $5-8$ & Natural & MK 906 \\
\hline $\begin{array}{l}\text { Trachystemon orientalis (L.) G. Don } \\
\text { Brassicaceae }\end{array}$ & Kaldirik & $\mathrm{Cr}$ & $3-5$ & Natural & MK 951 \\
\hline $\begin{array}{l}\text { Barbarea vulgaris } \mathrm{R} . \text { Br. subsp. } \\
\text { vulgaris }\end{array}$ & Nicarotu & $\mathrm{H}$ & $4-5$ & Natural & MK 826 \\
\hline Brassica oleracea $\mathrm{L}$. & Lahana & $\mathrm{H}$ & $5-6$ & Cultivation & MK 831 \\
\hline $\begin{array}{l}\text { Cardamine impatiens L. subsp. } \\
\text { impatiens }\end{array}$ & Sultan kodimotu & $\mathrm{Th}$ & $6-8$ & Natural & MK 837 \\
\hline C. quinquefolia (M. Bieb.) Schmalh. & Hanımgömleği & $\mathrm{H}$ & $3-5$ & Natural & MK 838 \\
\hline $\begin{array}{l}\text { Eruca vesicaria }(\text { L.) Cav. } \\
\text { Caprifoliaceae }\end{array}$ & Roka & Th & $3-5$ & Cultivation & MK 868 \\
\hline Lonicera japonica Thunb. & Japon hanımeli & $\mathrm{Ph}$ & $4-7$ & Naturalized & MK 894 \\
\hline L.orientalis Lam. & Has çakkana & $\mathrm{Ph}$ & $5-7$ & Natural & MK 895 \\
\hline $\begin{array}{l}\text { Weigela floribunda C.A. Mey. } \\
\text { Cistaceae }\end{array}$ & Gelin tac1 & $\mathrm{Ph}$ & $5-6$ & Ornamental & MK 963 \\
\hline Cistus creticus L. & Laden & $\mathrm{Ph}$ & $3-6$ & Natural & MK 844 \\
\hline $\begin{array}{l}\text { C. salviifolius } \mathrm{L} \text {. } \\
\text { Commelinaceae }\end{array}$ & Kartli & $\mathrm{Ph}$ & $3-5$ & Natural & MK 845 \\
\hline $\begin{array}{l}\text { Tradescantia fluminensis Vell. } \\
\text { Convolvulaceae }\end{array}$ & Ak telgrafçiçeği & $\mathrm{Cr}$ & $5-9$ & Naturalized & MK 952 \\
\hline Calystegia sylvatica (Kit.) Griseb. & Bürük & $\mathrm{H}$ & $4-8$ & Natural & MK 835 \\
\hline $\begin{array}{l}\text { Convolvulus arvensis } \mathrm{L} . \\
\text { Cornaceae }\end{array}$ & Tarla sarmaşığı & $\mathrm{H}$ & $4-9$ & Natural & MK 847 \\
\hline Cornus mas L. & Kizılcık & $\mathrm{Ph}$ & $4-5$ & Natural & MK 848 \\
\hline Cucurbitaceae & & & & & \\
\hline Cucumis sativus L. & Hiyar & Th & $7-9$ & Cultivation & MK 854 \\
\hline Cucurbita pepo L. & Sakız kabağı & Th & $7-8$ & Cultivation & MK 853 \\
\hline $\begin{array}{l}\text { Sicyos angulatus L. } \\
\text { Ebenaceae }\end{array}$ & İtdolanbacı & $\mathrm{Th}$ & $7-10$ & Naturalized & MK 942 \\
\hline $\begin{array}{l}\text { Diospyros lotus L. } \\
\text { Ericaceae }\end{array}$ & Hirnık & $\mathrm{Ph}$ & $5-6$ & Natural & MK 860 \\
\hline Arbutus andrachne L. & Sandal ağacı & $\mathrm{Ph}$ & $3-5$ & Natural & MK 824 \\
\hline Calluna vulgaris (L.) Hull & Süpürge çalısı & $\mathrm{Ph}$ & $8-10$ & Natural & MK 834 \\
\hline Erica arborea $\mathrm{L}$. & Funda & $\mathrm{Ph}$ & $3-7$ & Natural & MK 865 \\
\hline Rhododendron luteum Sweet & Zifin & $\mathrm{Ph}$ & $4-9$ & Natural & MK 927 \\
\hline R. ponticum $\mathrm{L}$. & Kumar & $\mathrm{Ph}$ & $3-8$ & Natural & MK 928 \\
\hline Vaccinium arctostaphylos L. & Likarpa & $\mathrm{Ph}$ & $5-7$ & Natural & MK 957 \\
\hline
\end{tabular}


Table 2 (Contd.)

\begin{tabular}{|c|c|c|c|c|c|}
\hline Name of the taxa & Turkish name & $\begin{array}{l}\text { Life } \\
\text { form }\end{array}$ & $\begin{array}{l}\text { Flowering } \\
\text { period }\end{array}$ & Status & $\begin{array}{l}\text { Voucher } \\
\text { specimen }\end{array}$ \\
\hline \multicolumn{6}{|l|}{ Fagaceae } \\
\hline $\begin{array}{l}\text { Castanea sativa Mill. } \\
\text { Geraniaceae }\end{array}$ & Kestane & $\mathrm{Ph}$ & $6-7$ & Natural & MK 839 \\
\hline $\begin{array}{l}\text { Geranium asphodeloides Burm. f. } \\
\text { subsp. asphodeloides }\end{array}$ & Yaramerhemi & $\mathrm{H}$ & $4-6$ & Natural & MK 872 \\
\hline G. pusillum Burm. f. & İncegelinçarşafi & Th & $5-6$ & Natural & MK 873 \\
\hline G. robertianum L. & Dağ 1 tırı & Th & $4-6$ & Natural & MK 874 \\
\hline \multicolumn{6}{|l|}{ Hydrangeaceae } \\
\hline Deutzia gracilis Siebold \& Zucc. & Havlu püskülü & $\mathrm{Ph}$ & $4-6$ & Ornamental & MK 858 \\
\hline Hydrangea macrophylla (Thunb.) Ser. & Ortanca & $\mathrm{Ph}$ & $5-6$ & Ornamental & MK 879 \\
\hline $\begin{array}{l}\text { Philadelphus coronarius L. } \\
\text { Hypericaceae }\end{array}$ & Filbahri & $\mathrm{Ph}$ & $5-6$ & Ornamental & MK 911 \\
\hline \multicolumn{6}{|l|}{ Iridaceae } \\
\hline Iris lazica Albov & Laz süseni & $\mathrm{Cr}$ & $2-4$ & Natural & MK 882 \\
\hline I. pseudacorus L. & Bataklı süseni & $\mathrm{Cr}$ & $4-5$ & Natural & MK 883 \\
\hline $\begin{array}{l}\text { Iris } \times \text { germanica } \mathrm{L} \text {. } \\
\text { Juglandaceae }\end{array}$ & Göksüsen & $\mathrm{Cr}$ & $4-5$ & Ornamental & MK 881 \\
\hline \multicolumn{6}{|l|}{ Lamiaceae } \\
\hline Ajuga orientalis $\mathrm{L}$. & Dağmayasılı & $\mathrm{H}$ & $4-7$ & Natural & MK 821 \\
\hline Lamium album L. subsp. album & Balıcak & $\mathrm{H}$ & $5-8$ & Natural & MK 885 \\
\hline $\begin{array}{l}\text { L. galeobdolon (L.) L. subsp. } \\
\text { galeobdolon }\end{array}$ & Sarı balıcak & $\mathrm{H}$ & $4-6$ & Natural & MK 886 \\
\hline L. purpureum L. subsp. purpureum & Ballibaba & Th & $3-5$ & Natural & MK 887 \\
\hline Prunella vulgaris L. & Gelinciklemeotu & $\mathrm{H}$ & $5-9$ & Natural & MK 920 \\
\hline Melissa officinalis L. subsp. officinalis & Oğulotu & $\mathrm{H}$ & $6-7$ & Natural & MK 901 \\
\hline $\begin{array}{l}\text { Mentha longifolia (L.) L. subsp. } \\
\text { longifolia }\end{array}$ & Pünk & $\mathrm{H}$ & $6-8$ & Natural & MK 902 \\
\hline M. pulegium $\mathrm{L}$. & Yarpuz & $\mathrm{H}$ & $6-9$ & Natural & MK 903 \\
\hline $\begin{array}{l}\text { Origanum vulgare L. subsp. } \\
\text { viridulum (Martrin-Donos) Nyman }\end{array}$ & Karakınık & $\mathrm{H}$ & $5-10$ & Natural & MK 907 \\
\hline Rosmarinus officinalis L. & Biberiye & $\mathrm{Ph}$ & $2-5$ & Natural & MK 932 \\
\hline $\begin{array}{l}\text { Salvia verticillata } \mathrm{L} \text {. subsp. } \\
\text { verticillata }\end{array}$ & Dadırak & $\mathrm{H}$ & $6-8$ & Natural & MK 938 \\
\hline Stachys sylvatica $\mathrm{L}$. & Hamisirgan & $\mathrm{H}$ & $6-9$ & Natural & MK 947 \\
\hline \multicolumn{6}{|l|}{ Lauraceae } \\
\hline Laurus nobilis L. & Defne & $\mathrm{Ph}$ & $3-5$ & Natural & MK 891 \\
\hline \multicolumn{6}{|l|}{ Leguminosae } \\
\hline Acacia dealbata Link. & Gümüşi akasya & $\mathrm{Ph}$ & $2-4$ & Ornamental & MK 817 \\
\hline Albizia julibrissin Durazz. & Gülibrişim & $\mathrm{Ph}$ & $7-8$ & Ornamental & MK 822 \\
\hline $\begin{array}{l}\text { Cercis siliquastrum L. subsp. } \\
\text { siliquastrum }\end{array}$ & Erguvan & $\mathrm{Ph}$ & $4-5$ & Natural & MK 841 \\
\hline $\begin{array}{l}\text { Lathyrus laxiflorus (Desf.) O. Kuntze } \\
\text { subsp. laxiflorus }\end{array}$ & Deli burçak & $\mathrm{H}$ & $5-8$ & Natural & MK 889 \\
\hline Lotus corniculatus L. var. tenuifolius L. & Gazalboynuzu & $\mathrm{H}$ & $4-9$ & Natural & MK 896 \\
\hline
\end{tabular}


Table 2. (Contd.)

\begin{tabular}{|c|c|c|c|c|c|}
\hline Name of the taxa & Turkish name & $\begin{array}{l}\text { Life } \\
\text { form }\end{array}$ & $\begin{array}{l}\text { Flowering } \\
\text { period }\end{array}$ & Status & $\begin{array}{l}\text { Voucher } \\
\text { specimen }\end{array}$ \\
\hline Medicago sativa L. subsp. sativa & Karayonca & $\mathrm{H}$ & $4-9$ & Natural & MK 899 \\
\hline Melilotus officinalis (L.) Desr. & Kokulu yonca & Th & $5-9$ & Natural & MK 900 \\
\hline Phaseolus vulgaris $\mathrm{L}$. & Fasulye & Th & $4-5$ & Cultivation & MK 910 \\
\hline Pisum sativum L. & Bezelye & Th & $4-5$ & Cultivation & MK 914 \\
\hline Robinia hispida $\mathrm{L}$. & K1ll1 akasya & $\mathrm{Ph}$ & 4-7 & Ornamental & MK 930 \\
\hline R. pseudoacacia $\mathrm{L}$. & Yalancı akasya & $\mathrm{Ph}$ & $4-6$ & Naturalized & MK 929 \\
\hline Trifolium campestre Schreb. & Üçgül & Th & $2-4$ & Natural & MK 953 \\
\hline T. pratense $\mathrm{L}$. var. pratense & Çayır üçgülü & $\mathrm{H}$ & $5-9$ & Natural & MK 955 \\
\hline T. repens $\mathrm{L}$. var. repens & Ak üçgül & $\mathrm{H}$ & $3-9$ & Natural & MK 954 \\
\hline Securigera varia (L.) Lassen & Körigen & $\mathrm{H}$ & $5-8$ & Natural & MK 941 \\
\hline Vicia cracca L. subsp. stenophylla Vel. & Meşe fiği & $\mathrm{H}$ & 5-7 & Natural & MK 961 \\
\hline Wisteria sinensis (Sims) Sweet & Çin mor salkımı & $\mathrm{Ph}$ & 4-7 & Ornamental & MK 964 \\
\hline \multicolumn{6}{|l|}{ Lythraceae } \\
\hline Lythrum salicaria $\mathrm{L}$. & Hevhulma & $\mathrm{H}$ & 6-8 & Natural & MK 897 \\
\hline \multicolumn{6}{|l|}{ Malvaceae } \\
\hline Malva sylvestris $\mathrm{L}$. & Ebegümeci & $\mathrm{H}$ & $5-10$ & Natural & MK 898 \\
\hline $\begin{array}{l}\text { Tilia rubra DC. subsp. caucasica } \\
\text { (Rupr.) V. Engl. }\end{array}$ & Kafkas ihlamuru & $\mathrm{Ph}$ & $6-7$ & Natural & MK 950 \\
\hline \multicolumn{6}{|l|}{ Myrtaceae } \\
\hline $\begin{array}{l}\text { Callistemon viminalis (Sol. ex } \\
\text { Gaertn.) G. Don ex Loudon }\end{array}$ & Firça çalısı & $\mathrm{Ph}$ & $4-6$ & Ornamental & MK 833 \\
\hline $\begin{array}{l}\text { Leptospermum scoparium J.R. Forst. } \\
\& \text { G. Forst. }\end{array}$ & Okyanus mersini & $\mathrm{Ph}$ & $5-9$ & Ornamental & MK 892 \\
\hline \multicolumn{6}{|l|}{ Oleaceae } \\
\hline Ligustrum japonicum Thunb. & Lügüstrüm & $\mathrm{Ph}$ & $5-6$ & Ornamental & MK 893 \\
\hline Syringa vulgaris $\mathrm{L}$. & Leylak & $\mathrm{Ph}$ & $5-6$ & Ornamental & MK 948 \\
\hline \multicolumn{6}{|l|}{ Onagraceae } \\
\hline \multicolumn{6}{|l|}{$\begin{array}{l}\text { Epilobium angustifolium } \mathrm{L} \text {. } \\
\text { Oxalidaceae }\end{array}$} \\
\hline Oxalis articulata Savigny & Pembe ekşiyonca & $\mathrm{H}$ & 6-9 & Ornamental & MK 909 \\
\hline \multicolumn{6}{|l|}{ Pinaceae } \\
\hline Picea orientalis (L.) Peterm. & Doğu ladini & $\mathrm{Ph}$ & $4-5$ & Natural & MK 912 \\
\hline $\begin{array}{l}\text { Pittosporaceae } \\
\text { Pittosporum tobira (Thunb.) W.T. } \\
\text { Aiton }\end{array}$ & Yıldız çalısı & $\mathrm{Ph}$ & $4-6$ & Ornamental & MK 915 \\
\hline \multicolumn{6}{|l|}{ Plantaginaceae } \\
\hline Plantago lanceolata $\mathrm{L}$. & Damarlica & $\mathrm{H}$ & $4-10$ & Natural & MK 916 \\
\hline $\begin{array}{l}\text { Veronica beccabunga L. subsp. } \\
\text { beccabunga }\end{array}$ & At teresi & $\mathrm{Cr}$ & $5-10$ & Natural & MK 959 \\
\hline$V$. persica Poir. & Cırcamuk & Th & $1-12$ & Natural & MK 960 \\
\hline
\end{tabular}


Table 2 (Contd.)

\begin{tabular}{|c|c|c|c|c|c|}
\hline Name of the taxa & Turkish name & $\begin{array}{l}\text { Life } \\
\text { form }\end{array}$ & $\begin{array}{l}\text { Flowering } \\
\text { period }\end{array}$ & Status & $\begin{array}{l}\text { Voucher } \\
\text { specimen }\end{array}$ \\
\hline \multicolumn{6}{|l|}{ Poaceae } \\
\hline Zea mays L. subsp. mays & Misir & Th & $6-10$ & Cultivation & MK 965 \\
\hline \multicolumn{6}{|l|}{ Primulaceae } \\
\hline Primula acaulis (L.) L. subsp. acaulis & Çuhaçiçeği & $\mathrm{H}$ & $3-6$ & Natural & MK 918 \\
\hline $\begin{array}{l}P . \text { acaulis }(\mathrm{L} .) \text { L. subsp. rubra }(\mathrm{Sm} .) \\
\text { Greuter \& Burdet }\end{array}$ & Evvelbahar çiçeği & $\mathrm{H}$ & $3-5$ & Natural & MK 919 \\
\hline \multicolumn{6}{|l|}{ Ranunculaceae } \\
\hline Helleborus orientalis Lam. & Çöpleme & $\mathrm{H}$ & $3-5$ & Natural & MK 877 \\
\hline $\begin{array}{l}\text { Ranunculus constantinopolitanus } \\
\text { (DC.) d'Urv }\end{array}$ & Kâğıthane çiçeği & $\mathrm{H}$ & $5-6$ & Natural & MK 924 \\
\hline $\begin{array}{l}\text { R. ficaria L. subsp. bulbifera } \\
\text { Lawalrée }\end{array}$ & Buğdaycık & $\mathrm{Cr}$ & $3-4$ & Natural & MK 925 \\
\hline$R$. repens $\mathrm{L}$. & Tiktakdana & $\mathrm{H}$ & $5-7$ & Natural & MK 926 \\
\hline \multicolumn{6}{|l|}{ Rosaceae } \\
\hline Cerasus avium (L.) Moench & Kiraz & $\mathrm{Ph}$ & $3-5$ & Cultivation & MK 840 \\
\hline Cydonia oblonga Mill. & Ayva & $\mathrm{Ph}$ & $5-6$ & Cultivation & MK 855 \\
\hline Duchesnea indica (Andrews) Focke & Sabun çileği & $\mathrm{H}$ & $5-9$ & Naturalized & MK 861 \\
\hline Eriobotrya japonica (Thunb.) Lindl. & Yenidünya & $\mathrm{Ph}$ & $10-1$ & Cultivation & MK 867 \\
\hline Fragaria vesca $\mathrm{L}$ & Dağ çileği & $\mathrm{H}$ & $4-6$ & Natural & MK 871 \\
\hline Laurocerasus officinalis M. Roem. & Karayemiş & $\mathrm{Ph}$ & $4-6$ & Natural & MK 890 \\
\hline Potentilla reptans $\mathrm{L}$. & Reşatınotu & $\mathrm{H}$ & $5-8$ & Natural & MK 917 \\
\hline $\begin{array}{l}\text { Prunus divaricata Ledeb. var. } \\
\text { divaricata }\end{array}$ & Yunus eriği & $\mathrm{Ph}$ & $4-5$ & Natural & MK 921 \\
\hline Pyracantha coccinea M. Roem. & Ateşdikeni & $\mathrm{Ph}$ & $4-6$ & Natural & MK 922 \\
\hline Pyrus communis L. subsp. communis & Armut & $\mathrm{Ph}$ & $4-5$ & Cultivation & MK 923 \\
\hline Rosa canina $\mathrm{L}$ & Kuşburnu & $\mathrm{Ph}$ & $5-7$ & Natural & MK 931 \\
\hline $\begin{array}{l}\text { Rubus canescens DC. var. glabratus } \\
\text { (Godr.) Davis \& Meikle }\end{array}$ & Çobankösteği & $\mathrm{Ph}$ & $5-8$ & Natural & MK 933 \\
\hline R. idaeus L. subsp. idaeus & Ahududu & $\mathrm{Ph}$ & $6-7$ & Natural & MK 934 \\
\hline R. sanctus Schreb. & Böğ̈̈rtlen & $\mathrm{Ph}$ & $6-8$ & Natural & MK 935 \\
\hline $\begin{array}{l}\text { Spiraea vanhouttei (Briot) Carrière } \\
\text { Rutaceae }\end{array}$ & İspirya & $\mathrm{Ph}$ & $4-5$ & Ornamental & MK 946 \\
\hline $\begin{array}{l}\text { Citrus sinensis (L.) Osbeck } \\
\text { Salicaceae }\end{array}$ & Portakal & $\mathrm{Ph}$ & $4-6$ & Cultivation & MK 846 \\
\hline Salix alba L. subsp. alba & Ak sögüt & $\mathrm{Ph}$ & $4-5$ & Natural & MK 936 \\
\hline $\begin{array}{l}\text { S. caprea } \mathrm{L} \text {. } \\
\text { Sapindaceae }\end{array}$ & Sorgun & $\mathrm{Ph}$ & $4-5$ & Natural & MK 937 \\
\hline $\begin{array}{l}\text { Acer cappadocicum Gled. subsp. } \\
\text { cappadocicum }\end{array}$ & Beşparmak ağacı & $\mathrm{Ph}$ & $3-5$ & Natural & MK 819 \\
\hline $\begin{array}{l}\text { A. heldreichii Orph. ex Boiss. subsp. } \\
\text { trautvetteri (Medw.) A.E. Murray }\end{array}$ & Kafkas akçaağacı & $\mathrm{Ph}$ & $3-5$ & Natural & MK 818 \\
\hline $\begin{array}{l}\text { Scrophulariaceae } \\
\text { Verbascum gnaphalodes M. Bieb. }\end{array}$ & Uslu siŏırkuvruğu & $\mathrm{H}$ & $5-9$ & Natural & MK 958 \\
\hline
\end{tabular}


Table 2 (Contd.)

\begin{tabular}{llllll}
\hline Name of the taxa & Turkish name & $\begin{array}{l}\text { Life } \\
\text { form }\end{array}$ & $\begin{array}{l}\text { Flowering } \\
\text { period }\end{array}$ & Status & $\begin{array}{l}\text { Voucher } \\
\text { specimen }\end{array}$ \\
\hline $\begin{array}{l}\text { Simaroubaceae } \\
\begin{array}{l}\text { Ailanthus altissima } \text { (Mill.) Swingle } \\
\text { Violaceae }\end{array}\end{array}$ & Kokarağaç & Ph & $5-6$ & Naturalized & MK 820 \\
\begin{tabular}{l} 
Viola odorata L. \\
\hline
\end{tabular} & Kokulu menekşe & H & $4-5$ & Natural & MK 962 \\
\hline
\end{tabular}

$\mathrm{Ph}=$ Phanerophytes, $\mathrm{Ch}=$ Chamaephytes, $\mathrm{H}=$ Hemicryptophytes, $\mathrm{Th}=$ Therophytes, $\mathrm{Cr}=$ Cryptophytes .

The current status of honeybee plants in Espiye revealed that the identified 149 taxa were clustered into four different ecological groups comprising 69\% natural, 16\% ornamental, $10 \%$ cultivation and 5\% naturalized. The majority of species are natural (103 taxa) followed by the ornamental (23 taxa), cultivation (15 taxa) and naturalized ( 8 taxa). In addition, flowering period of the identified taxa has been determined. As seen in Figure 3, the flowering period for plants in the study area is mostly between April and August. In May, many plants can be seen in flowering state in the study area. Some important natural, ornamental and naturalized bee plant species of Espiye region are shown in Figures 4 and 5.

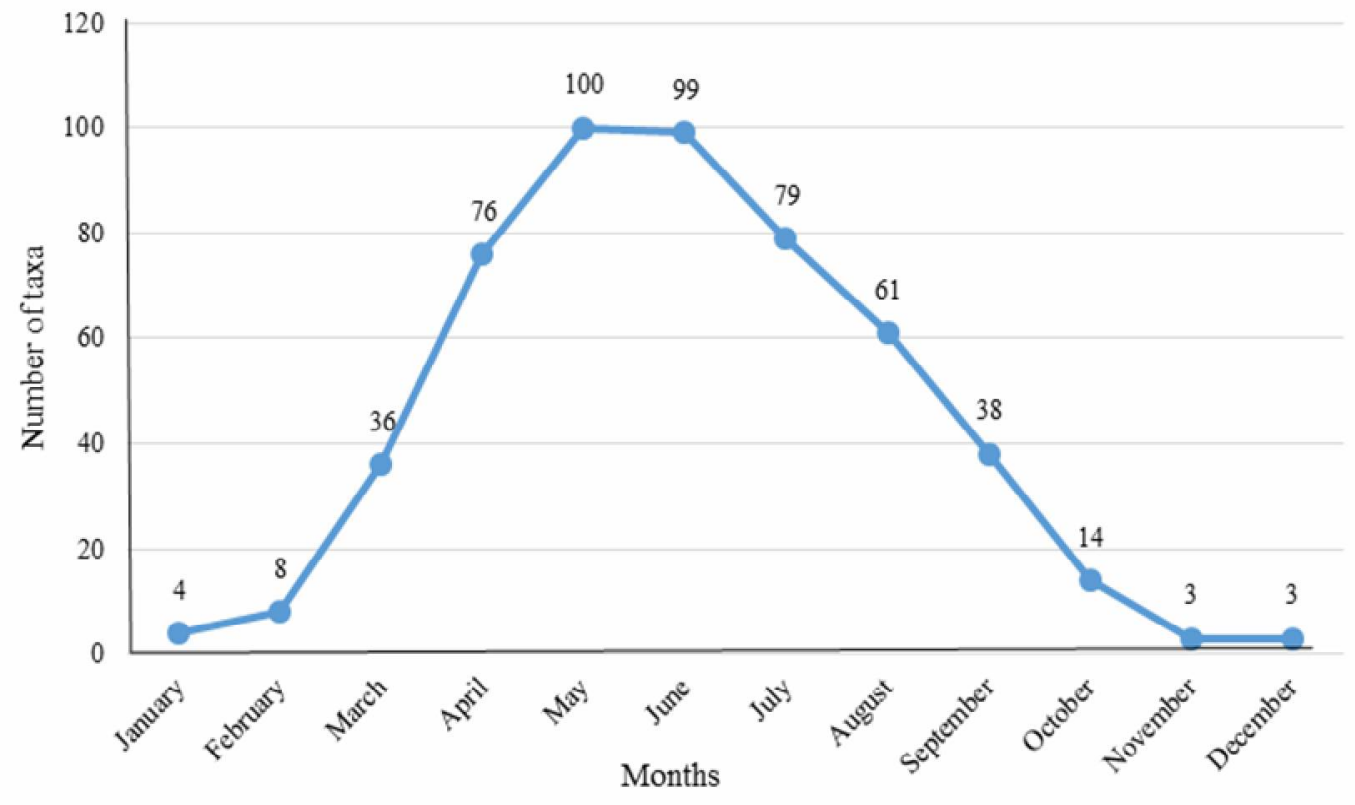

Fig. 3. Flowering period of the honeybee plants of Espiye, Giresun.

Honeybees cannot operate below $12-13^{\circ} \mathrm{C}$ (Korkmaz, 2015). Honeybees start to collect nectar and pollen in the month of May. Flowering in plants reach top level in Espiye in May and there is direct correlation between temperature, precipitation and flowering plants. Honeybee activity remains during May to October (Fig. 6). As seen in Figure 6, when flowering in plants accounts reach at the top level temperature is appropriate, and precipitation is at the least level. 

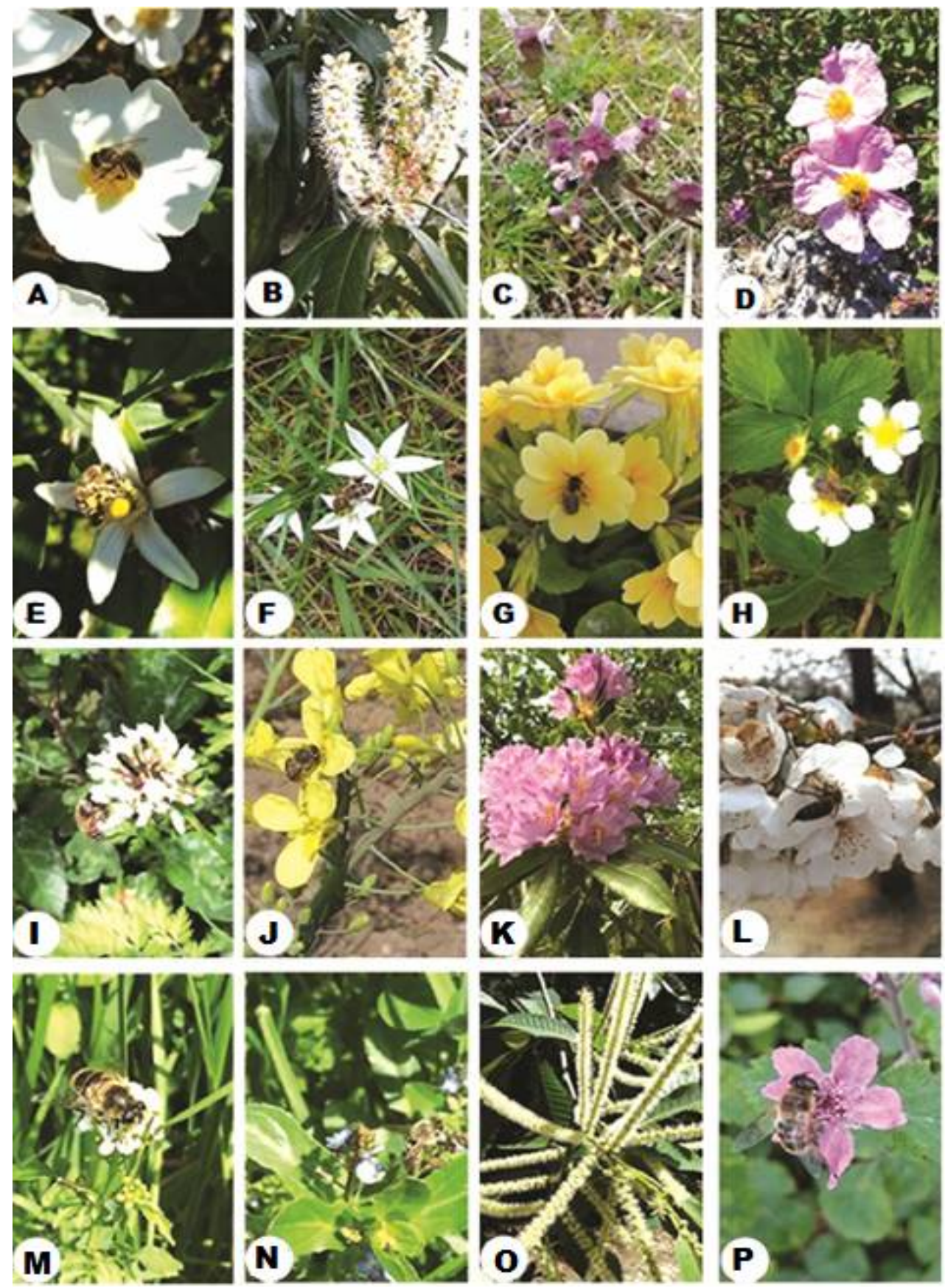

Fig. 4. Some important natural bee plants of Espiye region: A. Cistus salviifolius; B. Laurocerasus officinalis; C. Lamium purpureum; D. Cistus creticus; E. Citrus sinensis; F. Ornithogalum oligophyllum; G. Primula acaulis; H. Fragaria vesca; I. Trifolium repens; J. Brassica oleracea; K. Rhododendron ponticum; L. Cerasus avium; M. Cardamine impatiens; N. Veronica beccabunga; O. Castanea sativa; P. Rubus sanctus. 


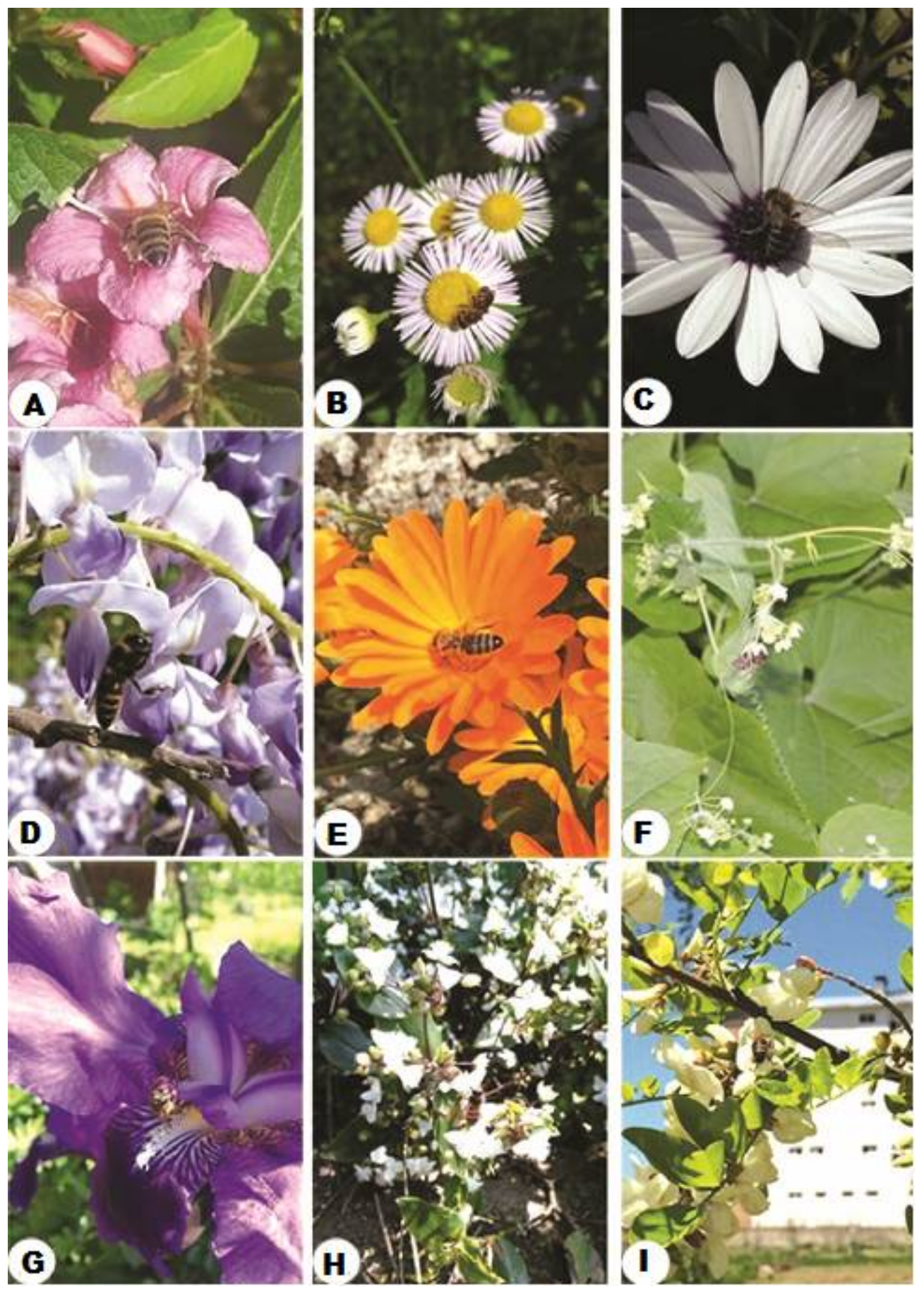

Fig. 5. Some important ornamental and naturalized bee plants of Espiye region: A. Weigela floribunda; B. Erigeron annuus; C. Dimorphotheca ecklonis; D. Wisteria sinensis; E. Calendula officinalis; F. Sicyos angulatus; G. Iris $\times$ germanica; H. Tradescantia fluminensis; I. Robinia pseudoacacia. 


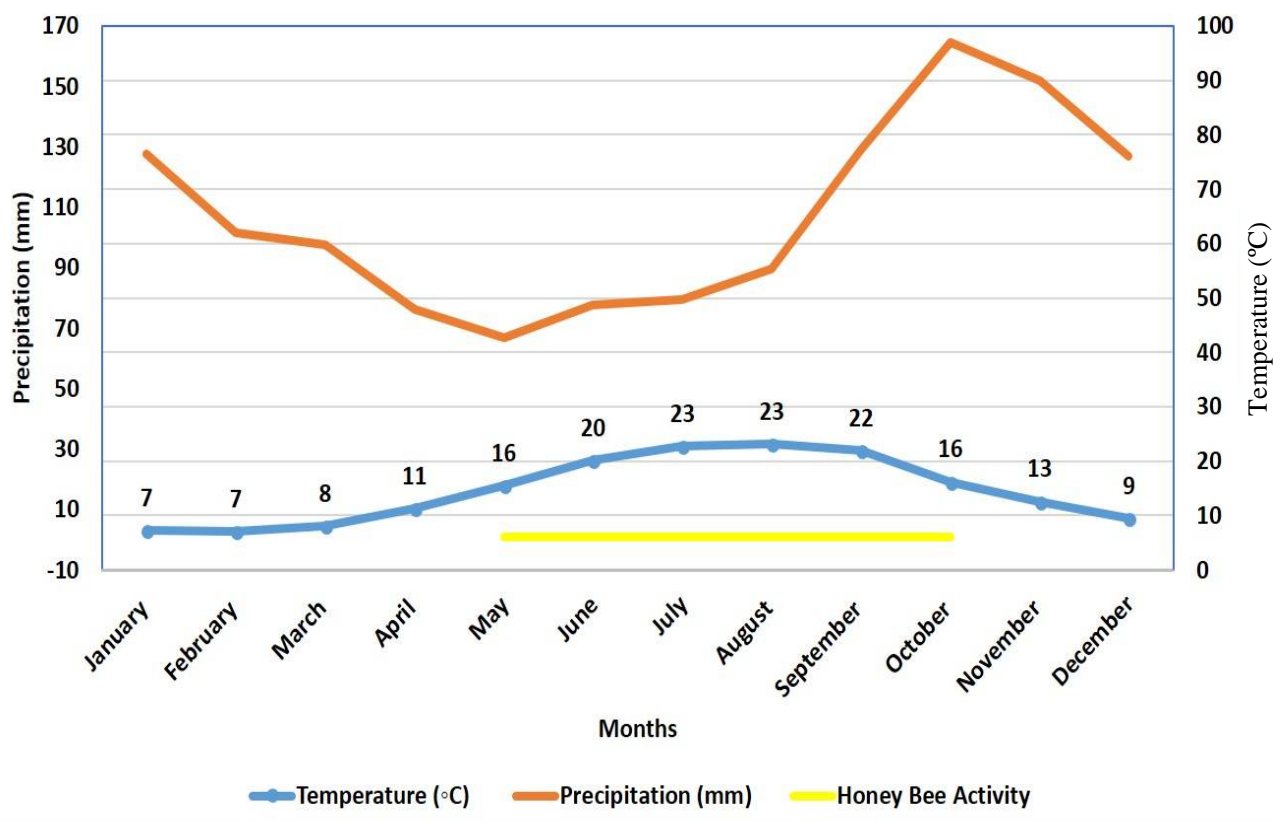

Fig. 6. Correlation between honeybee activity, temperature and precipitation.

In the present study 23 ornamental and eight naturalized plant species were identified as visited by the honeybees. The presence of these plants in ecosystems causes changes in the pollen and nectar resources of the honeybees. These changes have caused both the deterioration of the functions provided by the ecosystems and the decrease of the agricultural gains. In addition, this differentiation will also cause a change in the quality of the local honeys. Therefore, on the use of mostly local indigenous species there will be a significant share in the conservation of ecosystem balance.

\section{References}

Davis, P.H. (Ed.). 1965-1985. Flora of Turkey and the East Aegean Islands, Vols. 1-9. Edinburgh University Press, UK.

Davis, P.H, Mill, R.R. and Tan, K. 1988. Flora of Turkey and the East Aegean Islands. Vol. 10. Edinburgh University Press, UK.

Dokap, 2000. Dokap (Doğu Karadeniz Bölgesi Gelişme Planı), pp.1-9.

Duyum, S. and Friedman, S. 2015. The Turkish beekeeping and honey sector, Ankara, Report No. TR5021.

Güner, A., Özhatay, N. and Başer, K.H.C. 2000. Flora of Turkey and the East Aegean Islands, Vol. 11, Supplement-II. Edinburgh University Press, UK.

Güner, A., Aslan, S., Ekim, T., Vural, M. and Babaç, M.T. 2012. Türkiye bitkileri listesi (Damarlı bitkiler). Nezahat Gökyiğit Botanik Bahçesi ve Flora Araştırmaları Derneği Yayını, İstanbul.

Korkmaz, A. 2015. Bal Arısı Polinasyonu, Samsun Gıda Tarım ve Hayvancılık İl Müdürlügü Yayını, Samsun, p. 72.

OGM (Orman Genel Müdürlüğü). 2013-2015. Türkiye orman varlığı kitabı. Orman İdaresi ve Planlama Dairesi Başkanlığı, No. 115.

Özturk, F. and Erkan, C. 2010. Bee plants of Van Lake basin (Turkey). Int. J. Bot. 6: 101-106.

Raunkiaer, C. 1937. The Life Forms of Plants and Statistical Plant Geography. Clarendon Press, Oxford. 
Siral1, R. 2009. Important honey production regions of Turkey. J. Beekeeping Res. 1: 16-20.

Tew, J.E. 1998. Some Ohio Nectar and Pollen Production Plants. Ohio State University, Columbus.

TSMS. 2017. Turkish State Meteorological Service Bulletin.

Tucak, Z., Tucak, A., Puskadija, Z. and Tucak, M. 2000. Nutritious healing composition of some kinds of honey in Eastern Croatia. Agriculture 6: 129-132.

Tucak, Z., Perispic, M., Beslo, D. and Tucak, I. 2004. Influence of the beehive type on the quality of honey. Coll Antropol. 28: 463-467.

TUIKK. 2018. Turkish Statistical Institute. [Accessed on 07.02.2018]

Tutkun, E. 2000. Technical Beekeping Handbook. Development Foundation of Turkey, Ankara.

(Manuscript received on 12 February 2018; revised on 21 March 2018) 